Supporting Information

\title{
Nonthermal Plasma Synthesis of Gallium Nitride Nanoparticles: Implications for Optical and Electronic Applications
}

\author{
Alexander Hoํ․ Rajib Mandal ${ }^{1}$, Richard R. Lunt ${ }^{2}$, and Rebecca J. Anthony 1 ,2,a) \\ 1)Dept. of Mechanical Engineering, 428 S. Shaw Lane, Suite 2555, Michigan State University, East Lansing, MI \\ 48824 \\ 2)Dept. of Chemical Engineering and Materials Science, Michigan State University, 428 S. Shaw Lane, Suite 2100, \\ East Lansing, MI 48824
}

\section{Reactor Details}

For the synthesis of GaN nanoparticles in this work a nonthermal RF plasma reactor was used. A quartz tube with 0.375-inch outer diameter and 0.275-inch inner diameter was used for synthesis. Three gases were used during synthesis, argon, ammonia, and trimethylgallium. The ammonia served as the precursor gas for nitrogen and the trimethylgallium served as the precursor source for gallium, while argon acted as a background gas. Flow rates were regulated with mass flow controllers. Power delivery to the reactor was acheived with three ring electrodes placed concentrically with the reactor, a $13.56 \mathrm{MHz}$ RF power supply, and an impedance matching network. The top and bottom electrodes were separated by six inches and the middle electrode was centered between the top and bottom electrodes. The electrodes were attached to a matching network which was used to match the impedance of the power supply to the impedance of the plasma in order to maximize power delivery to the plasma.

\section{Measurement Techniques and Data Analysis}

Samples for X-ray diffraction were prepared by collecting particles in the reactor on a glass slide. A feedthrough into the reactor allows for a push rod to be inserted and rostered, which was used to form a layer of particles to for analysis. A Rigaku Smart $\mathrm{Lab}$ diffractometer with a Cu-Ko x-ray source was used to perform the measurements. A parallel beam incident optic was used to collimate the beam, the incident beam was also positioned at the critical angle of $\mathrm{GaN}$ to further enhance intensity from the sample. Matlab was used to post process data, specifically for fitting a curve to the experimental data and deconvolution of the peaks present in the diffraction pattern.

Particles for TEM were collected on steel meshes placed in line with the flow, directly downstream of the reactor. Meshes were then removed from the reactor and placed in tubes with ethanol which was then sonicated to remove particles form the mesh. Meshes were then removed from tube and particles were drop casted onto TEM grids. A JEOL 2200FS TEM was used for analysis. Following image collection samples were analyzed using ImageJ, particle diameters were estimated by profiling particles and approximating shape to be spherical to calculate particle diameter. Interplanar spacing was measured by identifying particles with clear crystal structure and plotting grayscale values, from which the average distance between minima and maxima was used to estimate interplanar spacing. 
The Tauc plot was made from the UV-VIS absorption spectrum of the GaN nanoparticles. Samples were dispersed into ethanol and then a quartz cuvette with a $10 \mathrm{~mm}$ path length was used during the measurement. A Matlab script was written to identify the linear portions of the Tauc plot and extrapolate line to the energy axis. The script computes the second derivative of the Tauc plot and finds where the second derivative is equal to zero. This is done to identify the locations where linear behavior in the Tauc plot is present. Once the zero locations of the second derivative are found tangent lines to the plot at those locations are graphed and the bandgap extrapolated from the position where the tangent line crosses the energy axis. Low energy regions in the plot $(\mathrm{E}<3.5 \mathrm{eV})$ exhibiting any linearity were excluded as possible locations to extrapolate the bandgap as these portions of the plot match with the exponential growth of the absorption coefficient consistent with the Urbach tail commonly observed in materials at sub-band gap energies. The analysis yielded several linear potions in the 5 to $6 \mathrm{eV}$ range, with bandgap extrapolations between 5.5 and $5.6 \mathrm{eV}$.

Samples for XPS were prepared by collecting particles in the reactor on a copper substrate. A feedthrough into the reactor allows for a push rod to be inserted and rostered, which was used to form a layer of particles to for analysis. XPS analysis was conducted on a Perkin Elmer Phi 5600 ESCA system. Matlab was used for further analysis of XPS spectra, primarily for deconvolution of induvial peaks present in the spectra.

Samples for FTIR were prepared by collecting particles in the reactor on a copper substrate. A feedthrough into the reactor allows for a push rod to be inserted and rostered, which was used to form a layer of particles to for analysis. IR spectra were collected immediately following synthesis and at several time intervals following deposition. A Bruker Alpha Fourier Transform Infrared spectrometer was used for analysis. Matlab was used for further analysis of FTIR spectra, primarily for deconvolution of induvial peaks present in the spectra.

Particles for EPR were collected on steel meshes placed in line with the flow, directly downstream of the reactor. Following synthesis, the mesh was removed and placed into a test tube where the sample was sonicated and lightly tapped to remove particles from the filter. Particles were then transferred from the test tube to an EPR for analysis. A Bruker ELEXSYS-II E580 EPR instrument was used to measure the EPR signal at room temperature. 

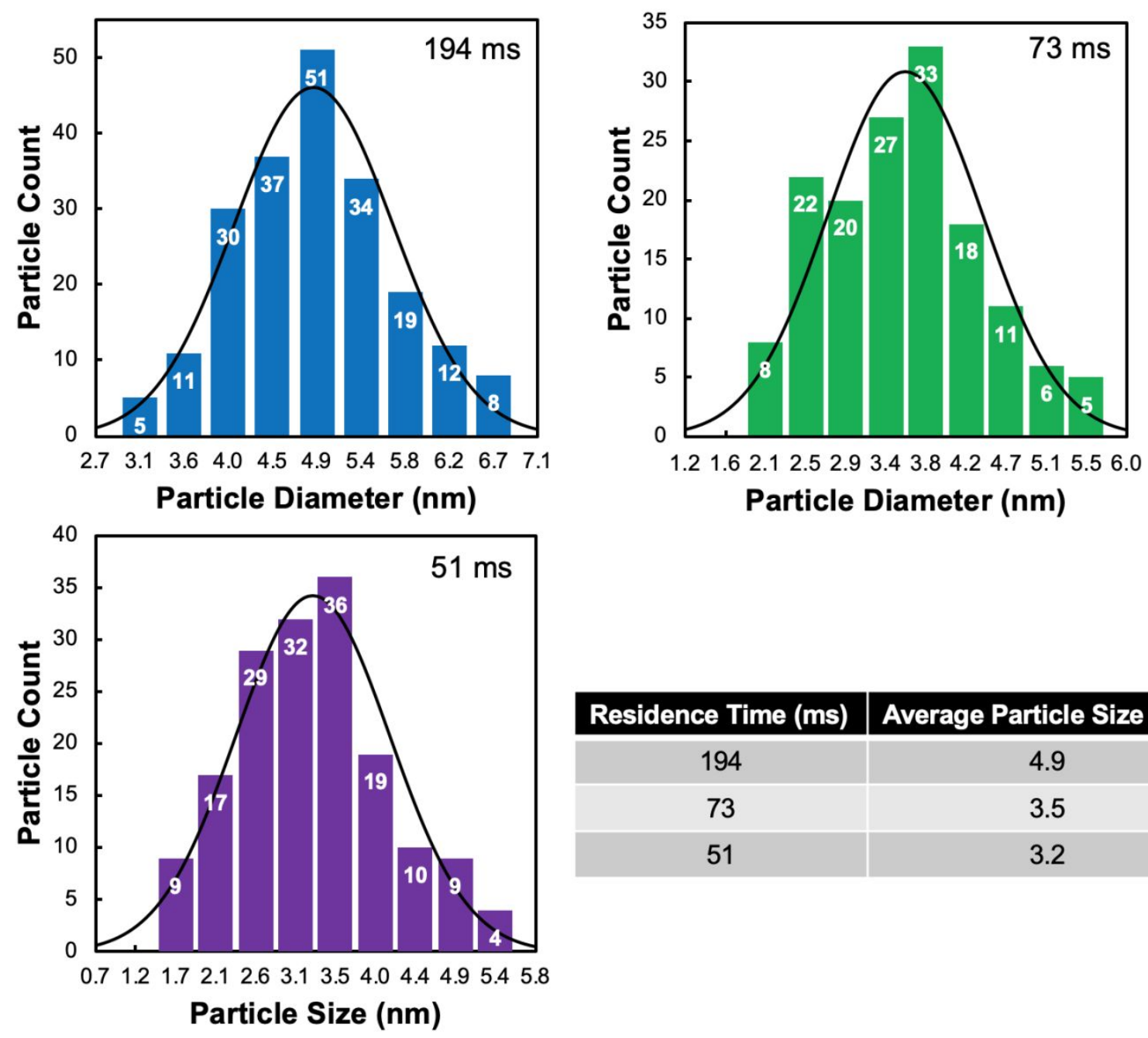

\section{Residence Time (ms) Average Particle Size (nm)}

\begin{tabular}{|c|c|c|}
\hline 194 & 4.9 \\
\hline 73 & 3.5 \\
\hline 51 & 3.2 \\
\hline
\end{tabular}

Figure S1: Particle size distributions at different residence times. Particle size disrbutions were measured by analysis of TEM images. The use of Image J software was used to analyze the TEM images. Profiles of particles were drawn, the area was calculated based on the profile, and then an average diameter was calculated based on the area. 


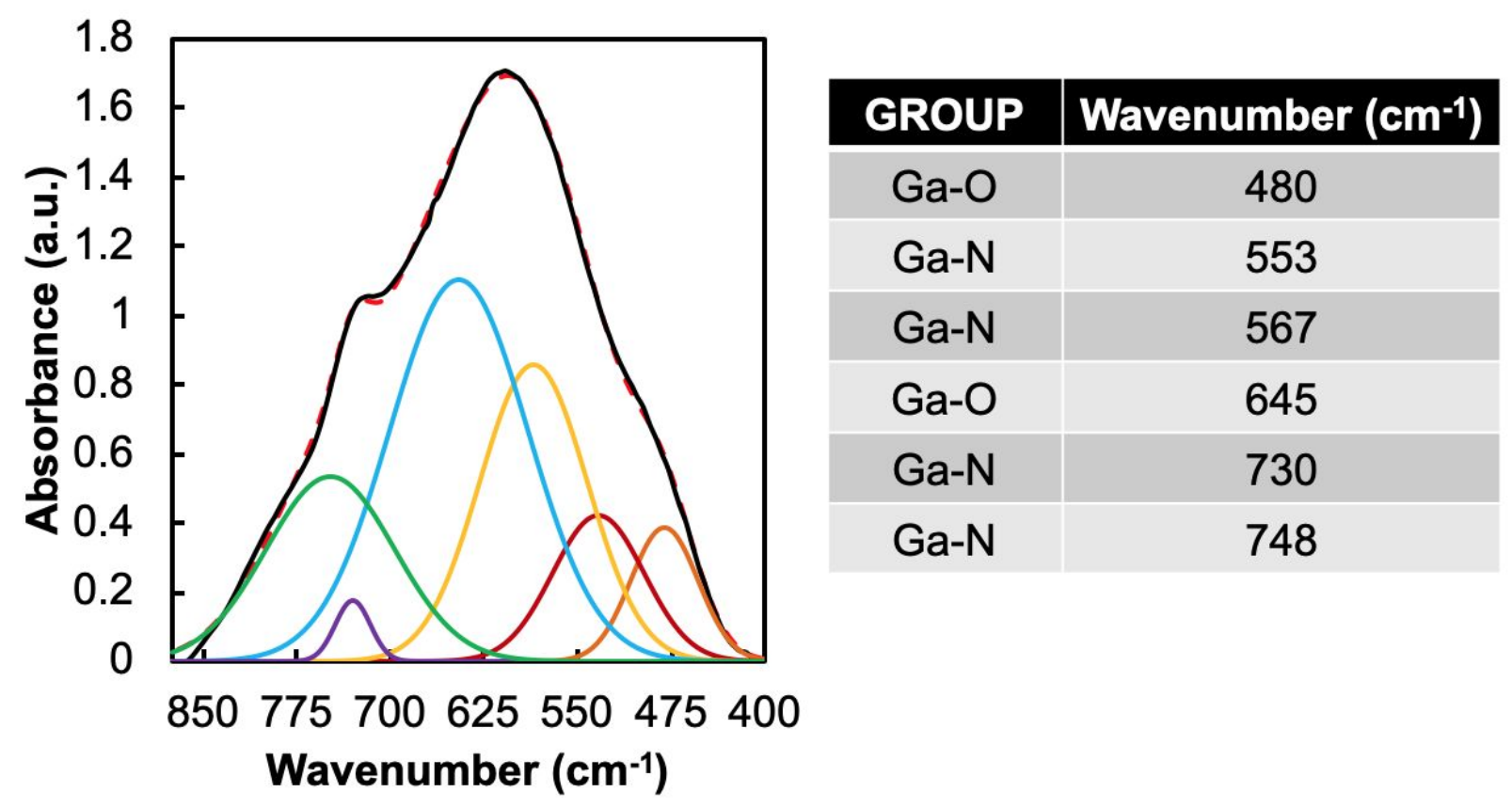

Figure S2: Portion of FTIR spectrum of GaN nanoparticles.

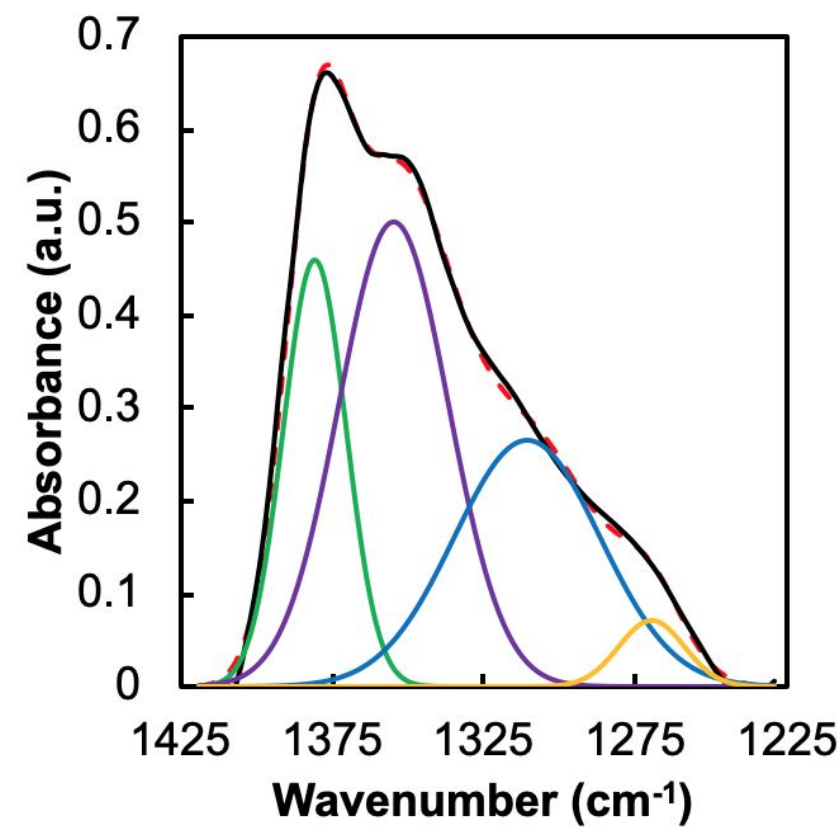

\section{GROUP Wavenumber $\left(\mathrm{cm}^{-1}\right)$}

$\mathrm{O}-\mathrm{H}$

1381

$\mathrm{O}-\mathrm{H}$

1355

C-O

1311

C-O

1270

Figure S3: Portion of FTIR spectrum of $\mathrm{GaN}$ nanoparticles. 


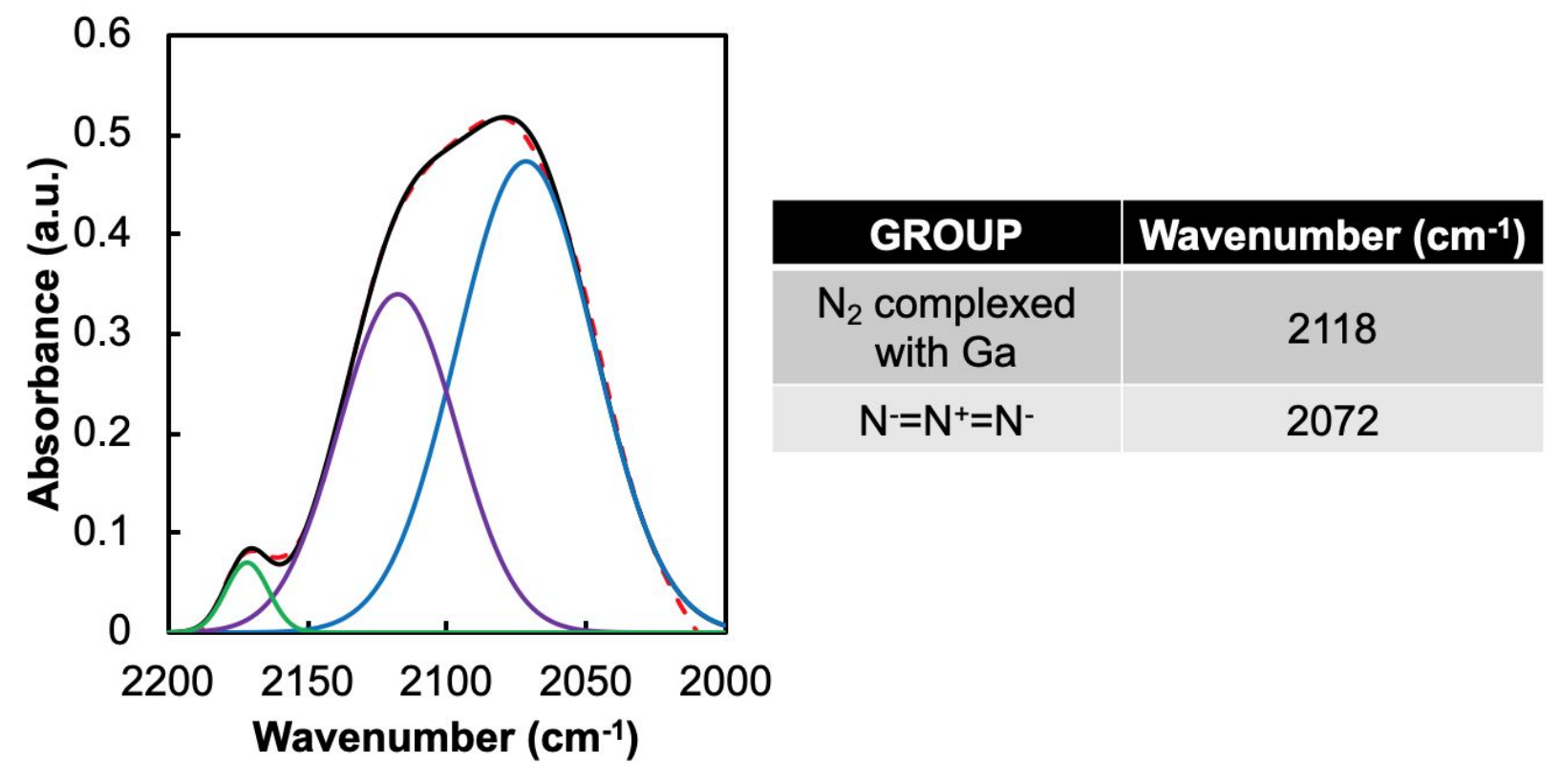

Figure S4: Portion of FTIR spectrum of GaN nanoparticles.

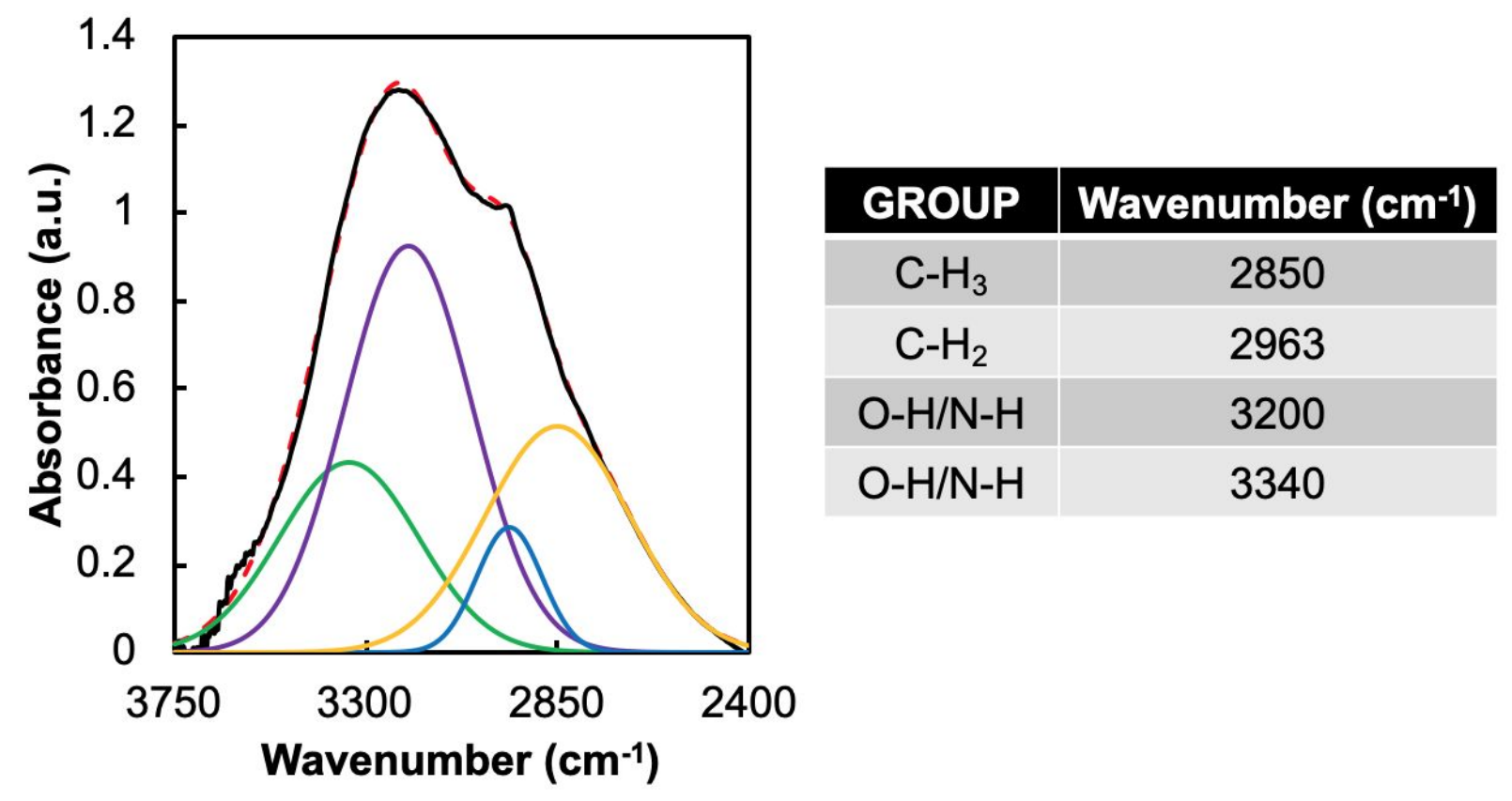

Figure S5: Portion of FTIR spectrum of GaN nanoparticles.

FTIR spectrum of GaN nanoparticles was collected on a copper substrate. Post processing of the data was conducted to determine the contribution of surface groups that contribute to the overall spectrum. Use of a MATLAB code was used to fit gaussian peaks to the spectrum. Intial peak positions and peak widths were provided as inputs, the best of 100 fits was then chosen. The goodness of fit was described with an $\mathrm{R}^{2}$ value. In all instances the $R^{2}$ value was greater than 0.99 . 